\title{
A Fuzzy Delay Approach for HIV Dynamics Using a Cellular Automaton
}

\author{
R. Motta Jafelice, ${ }^{1}$ C. A. F. Silva, ${ }^{2}$ L. C. Barros, ${ }^{3}$ and R. C. Bassanezi ${ }^{3}$ \\ ${ }^{1}$ Faculty of Mathematics, Federal University of Uberlândia, 38408-100 Uberlândia, MG, Brazil \\ ${ }^{2}$ Faculty of Mechanical Engineering, Federal University of Uberlândia, 38408-100 Uberlândia, MG, Brazil \\ ${ }^{3}$ Department of Applied Mathematics, IMECC, State University of Campinas, 13083-859 Campinas, SP, Brazil \\ Correspondence should be addressed to R. Motta Jafelice; rmotta@ufu.br
}

Received 3 March 2015; Revised 12 May 2015; Accepted 14 May 2015

Academic Editor: Jianming Zhan

Copyright (C) 2015 R. Motta Jafelice et al. This is an open access article distributed under the Creative Commons Attribution License, which permits unrestricted use, distribution, and reproduction in any medium, provided the original work is properly cited.

\begin{abstract}
The objective of this research is to study the evolution of CD4+ T lymphocytes infected with HIV in HIV-seropositive individuals under antiretroviral treatment utilizing a mathematical model consisting of a system of delay-differential equations. The infection rate of CD4+ T lymphocytes is a time-dependent parameter with delay. Such delay is given by a fuzzy number due to the uncertainty of the effects of both pharmacological and intracellular delays. A cellular automaton is utilized to estimate the parameters of the system. The effects of antiretroviral therapy in the cellular automaton are modeled using a fuzzy rule-based system with two inputs: the medication potency and the treatment adhesion for three hypothetical individuals. For each of them, we determine the infection rate of CD4+ T lymphocytes, which is different from zero, as opposed to other studies reported in the literature. As the infection rate is considered a fuzzy parameter, we determine the fuzzy and the defuzzified solutions for the infected CD4+ T lymphocytes. We obtain the maximum values of infected cells for individuals that receive low, medium, and high potency medication and treatment adhesion. The results obtained are in accordance qualitatively with what would be expected in a real situation.
\end{abstract}

\section{Introduction}

AIDS (acquired immunodeficiency syndrome) is an infectious and contagious disease caused by HIV (human immunodeficiency virus) which leads to a progressive loss of infected individuals' immunity. This syndrome is characterized by signals and symptoms which are due to the reduced rate of CD4+ $\mathrm{T}$ lymphocytes within the bloodstream which are cells of paramount importance for the perfect function of the human immune system. Since HIV was identified, AIDS became a world epidemic of great proportions. Experts from the United Nations estimate that in 2013 about 35 million people were contaminated worldwide with HIV. In Latin American countries 1.6 million people are infected, which indicates that they face a biologic problem of great social and economic repercussion [1]. The virus produces three proteins that are of paramount importance for the replication process: reverse transcriptase, integrase, and protease. When the virus reaches the bloodstream, it attacks mainly the CD4+ T lymphocytes. The quantity of $\mathrm{CD} 4+\mathrm{T}$ lymphocytes in peripheral blood presents important prognostic implication for the evolution of the viral infection. The antiretroviral treatment works through the inhibition of those three proteins. The inhibitors prevent free virus particles from infecting CD4+ T lymphocytes delaying viral replication and allowing the organism to react naturally. The inhibition of these three enzymes has led to a substantial improvement in HIV therapy [2].

In the last decades, fuzzy set theory has contributed significantly to the mathematical modeling of uncertain phenomena such as in the case of the infection by HIV [35]. Zarei et al. [6] proposed a fuzzy mathematical model of HIV infection which consisted of a linear fuzzy differential equation system that describes the level of immune cells and the viral load due to the inherent fuzziness of the immune system's strength. The authors also proposed a fuzzy optimal control problem that minimizes both the viral load and the systemic cost for the body.

The deterministic mathematical models described by delay-differential equations are sometimes more realistic than the ones with no delay. Herz et al. [7] developed a 
deterministic model to describe the behavior of uninfected and infected CD4+ T lymphocytes and the plasma HIV load incorporating pharmacological and intracellular delays in individuals under antiretroviral therapy. Such delays are defined as, respectively, the time span during which the drugs reach an effective concentration and the time span between cell infection and the production of new virus particles. They also demonstrate that intracellular delay may affect the magnitude of the range of HIV decline causing variability or uncertainty in the response to the therapy with protease inhibitors. In many cases the equation parameters are uncertain and such equations should be reinterpreted. Traditionally such uncertainty has been treated by means of statistical methodologies. Mittler et al. [8] assume that the delay is characterized by a probability distribution. The adoption of distributed delay produced good results in the study of HIV dynamics under treatment. The authors above provide simulations close to the biological information given by Herz et al. (1996) in which the different phases of decay of the virus after treatment with antiretrovirals are represented graphically.

Jafelice et al. [9], using the model proposed by Herz et al. [7], modeled the virus rate decline in function of the delay at the onset of the treatment. The delay was considered a fuzzy parameter; therefore, a fuzzy solution was determined for the decline of the virus.

Cellular automata allow simulation of complex nonlinear dynamic systems simultaneously in time and space. The state of each cell of the automaton is modified according to its own state and the states of the cells in its neighborhood. These states correlate amongst themselves through simple rules that imitate the biological and physical laws which rule the behavior of the system [10]. Jafelice et al. (2009) simulated a cellular automaton, shaped like a torus, in which artificially uninfected and infected CD4+ T lymphocytes, free virus particles, and virus-specific cytotoxic T lymphocytes (CTLs) that attack infected cells within an individual undergoing antiretroviral therapy coexist.

The objective of this research is to propose a model of the evolution of infected CD4+ T lymphocytes in HIVseropositive individuals under antiretroviral treatment. From a cellular automaton [11] it is possible to obtain an approximate function of the infection rate coherent with the HIV dynamic behavior. In this paper we study HIV dynamics by means of a system of differential equations with uncertain delay in three hypothetical individuals submitted to three levels of treatment adhesion and three medication potencies. The parameters and the infection rate values for this system are obtained from the cellular automaton. The infection rate values are adjusted by the least squares method. Afterward we determine the numeric solution of the fuzzy model considering the delay as a triangle fuzzy number. Finally, we attain the defuzzified numeric solution for this model for each one of the three individuals. A different aspect of our research is that we consider the infection rate as different from zero, whereas in the model by Herz et al. (1996), set for simplification, such rate was equal to zero. Considering the infection rate equal to zero implies that the antiretroviral treatments are $100 \%$ effective, a situation that does not occur in reality since there is no definitive control of AIDS.

\section{Methodology}

2.1. Basic Concepts of Fuzzy Set Theory. First we define a fuzzy subset $D$ of $X$ given from a function $\mu_{D}: X \rightarrow[0,1]$, called the membership function, which is associated with the fuzzy set $D$, where $X$ is a nonempty set. The value $\mu_{D}(x)$ represents the degree of membership of $x \in D$. A classic subset $D$ of $X$ is a particular fuzzy set for which the membership function is the characteristic function $\mathscr{X}_{D}: X \rightarrow\{0,1\}[12]$.

The $\alpha$-cuts of the fuzzy set $D$ are denoted by $[D]^{\alpha}$ and defined as $[D]^{\alpha}=\left\{x \in X, \mu_{D}(x) \geq \alpha\right\}, 0<\alpha \leq 1 ;[D]^{0}=$ $\overline{\operatorname{supp}(D)}$, where $\operatorname{supp}(D)=\left\{x \in X, \mu_{D}(x)>0\right\}$ is the support of $D$. A fuzzy set $D$ is called a fuzzy number when $X=\mathbb{R}$ and all $\alpha$-cuts of $D$ are nonempty, all $\alpha$-cuts of $D$ are closed intervals of $\mathbb{R}$, and the support of $D$ is bounded.

Secondly we remind the reader that fuzzy rule-based systems (FRBS) is a concept that plays a key role in fuzzy set theory. They are broadly used in model problems, control, and classification [13] and may be considered as "expert systems" due to their basic composition that includes an inference machine and a rule base, both under the direct influence of human expert knowledge [14]. Four components comprise a FBRS: an input processor that performs the fuzzification of the input data, a collection of nebulous rules called rule bases, an inference machine, and an output processor that provides a real number. Once rule bases of the type "If. . .then..." are established, a FRBS can be understood as a mapping between an input and an output of the formula $y=f(x), x \in R^{n}$ and $y \in R^{m}$.

Thirdly a concept used also in this paper is Zadeh's Extension Principle. Its role is to obtain images of fuzzy sets via classic functions.

Let $X$ and $Y$ be two nonempty universal sets and a function $f: X \rightarrow Y$. Given a fuzzy set $D \subset X$, the fuzzy set $\widehat{f}(D) \subset Y$, with membership function given by

$$
\mu_{\widehat{f}(D)}(y)= \begin{cases}\sup _{\{x: f(x)=y\}} \mu_{D}(x) & \text { if }\{x: f(x)=y\} \neq \phi, \\ 0 & \text { otherwise, }\end{cases}
$$

is called Zadeh's Extension Principle of $D$ by $f$. Note that $\widehat{f}(D)=f(D)$ if $D$ is a classical set of $X$.

2.2. Classic Models for HIV Dynamics. Herz et al. [7] present a model which incorporates an antiretroviral treatment that contains three time-dependent variables: the population of uninfected cells $n(t)$, the population of infected cells that produce virus $i(t)$, and the plasma viral load $v(t)$, the three of them dying at the respective rates of $a, b$, and $s$. In this model it is assumed that uninfected cells are continually produced by the human body at a constant influx $r$. The infected cells produce virus particles at rate $k(t)$. In order to incorporate the virus life cycle in the intracellular phase of the model, Herz et al. [7] assume a time delay $\tau$ for virus production after cell infection. This implies that the recruitment of virusproducing cells at time $t$ is given by the density of cells that were newly infected at time $t-\tau$ and are still alive at time $t$. Moreover, we assumed a constant death rate $\tilde{a}$ for infected but not yet virus-producing cells. The probability of survival 


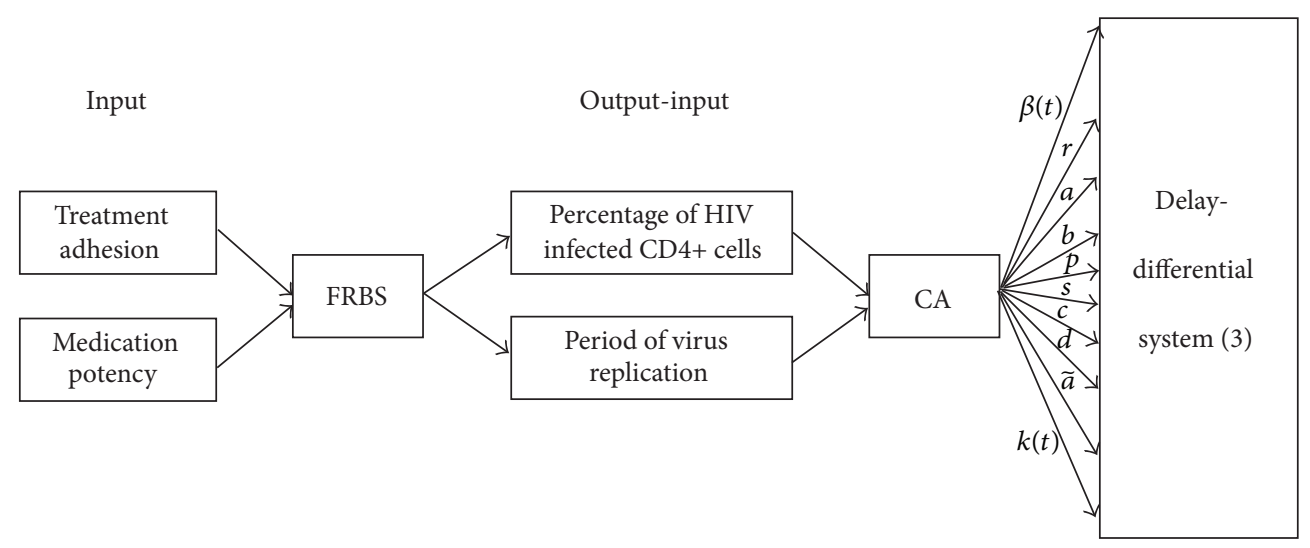

FIGURE 1: Schematic representation of the methodology utilized to obtain the parameters of system (3).

from $t-\tau$ to time $t$ is only $e^{-\widetilde{a} \tau}$. More generally, the probability of survival is given by a nonincreasing function $f(\tau)$ with $0 \leq f(\tau) \leq 1$. Thus, the model can be written as

$$
\begin{aligned}
& \frac{d n}{d t}=r-a n-\beta(t) n v, \\
& \frac{d i}{d t}=\beta(t-\tau) n(t-\tau) v(t-\tau) e^{-\widetilde{a} \tau}-b i \\
& \frac{d v}{d t}=k(t) i-s v .
\end{aligned}
$$

Based on the work by [15], we include in system (2) an equation for CTL as variable $z$ and a mortality rate piz caused by CTLs of infected cells in the second equation, as follows:

$$
\begin{aligned}
& \frac{d n}{d t}=r-a n-\beta(t) n v, \\
& \frac{d i}{d t}=\beta(t-\tau) n(t-\tau) v(t-\tau) e^{-\widetilde{a} \tau}-b i-p i z, \\
& \frac{d v}{d t}=k(t) i-s v, \\
& \frac{d z}{d t}=c i z-d z .
\end{aligned}
$$

The rate of CTL proliferation in response to antigen is ciz. In the absence of simulation CTLs decay at rate $d z$. The qualitative behavior of the HIV infection rate $\beta(t)$ in relation to the lymphocyte T CD4+ is also studied. The variables and parameters are dimensionless since the system of delaydifferential equations (3) is solved numerically according to the parameter values and initial conditions of the variables which are further described in Section 3.1.

In the next subsection we depict the cellular automaton and the FRBS used for attaining the parameters of the system.

2.3. Cellular Automaton and Fuzzy Rule-Based System. In the cellular automaton (CA) described by Jafelice et al. [11], uninfected and infected CD4+ T lymphocytes, free virus particles, and virus-specific CTL (cytotoxic T lymphocyte) coexist in the bloodstream of an individual under antiretroviral treatment. The CA is two-dimensional with Moore neighborhood [16] and periodic (toroidal) boundary [17]. The cells move in eight directions: north, east, south, west, northeast, northwest, southeast, and southwest. The changes in the states of the cells are implemented immediately; therefore the updating is asynchronous and at random. The CA model utilizes the output of the FRBS for the simulation.

The input variables of the FRBS are the adhesion to treatment and the medication potency (efficacy). The following linguistic values attributed to the variables are very low, low, medium, high, and very high. For adhesion to treatment, the interval $[0,1]$ is defined where 0 means none and 1 total adhesion. Based on [18] we define the medication potency interval as $[0.8,0.9]$. The output variables are the percentage of infected CD4+ T lymphocytes and the period of virus replication. For the former the linguistic terms are very low, low, medium, high, and very high and for the latter are very rapid, rapid, medium, slow, and very slow. For percentage of infected CD4+ T lymphocytes the interval is $[0.1,1]$ and for the period of virus replication is $[5,16]$. The lower limits of these two intervals correspond to the numbers of the iterations under the best treatment and the upper limits to the numbers of the iterations under no treatment [11]. The fuzzy rule base is constructed based on expert medical knowledge (Tables 1 and 2) utilizing the Mamdani inference method with centroid defuzzification [13].

As an example in Table 1 the first rule should be read as follows: "if medication potency is very low and adhesion is very low then the percentage of CD4+ cells that will be infected is very high."

Figure 1 depicts a diagram of the methodology used, in which we represent the FRBS input variable values (treatment adhesion and medication potency) and the FRBS output variable values (percentage of infected CD4+ T lymphocytes and the period of virus replication). The latter are the input values of the CA. From the simulations performed in the CA, the parameters of the delay-differential system (3) are determined.

At each iteration the number of HIV in the neighborhood of uninfected CD4+ T lymphocytes is counted. The product of this number by the output variable (percentage 

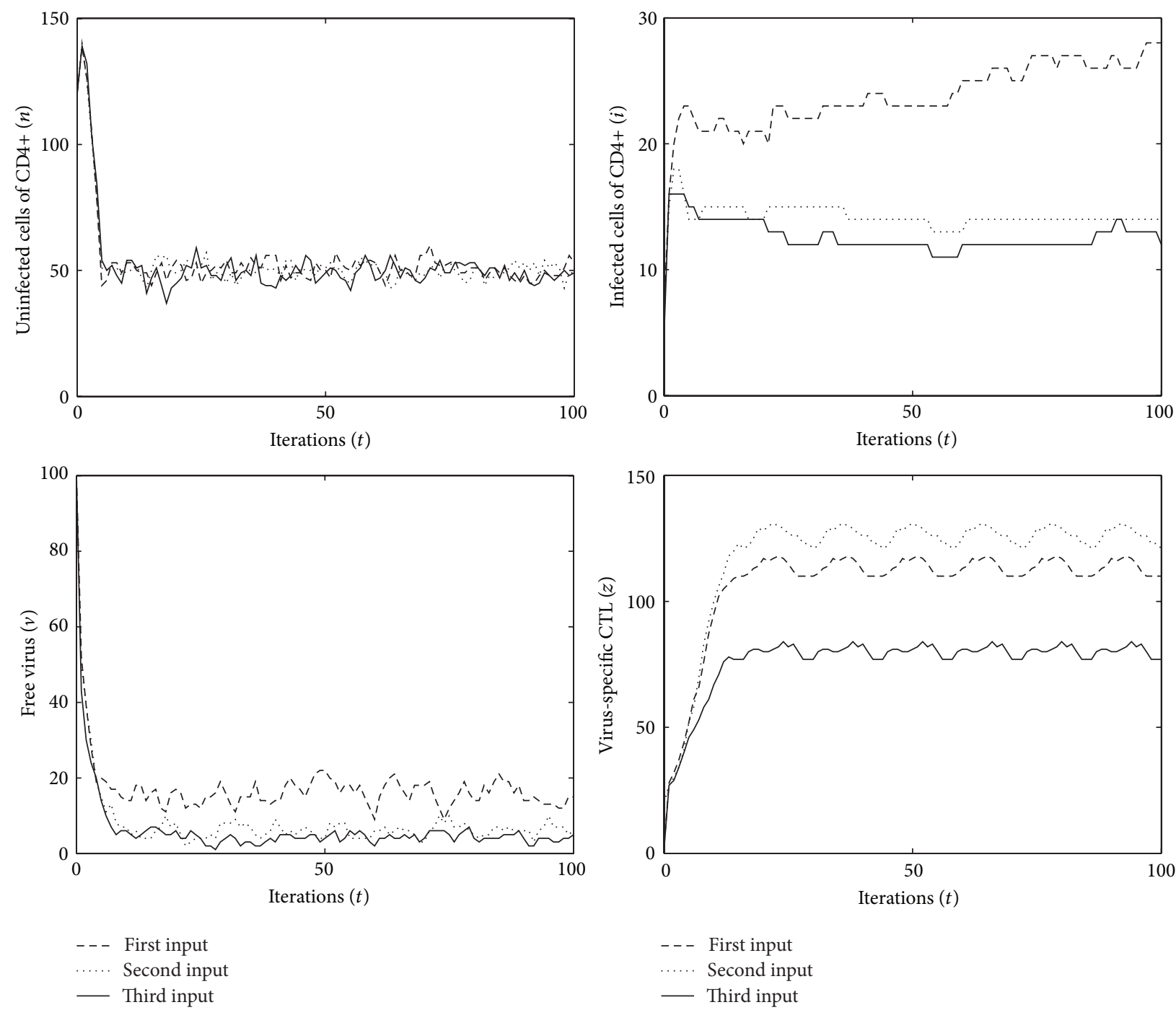

FIGURE 2: Results of cellular automaton simulation for the three individuals.

of CD4+ T lymphocytes) is the number of HIV infected cells. The simulation is performed using the values for treatment adhesion and medication potency of the three HIVseropositive hypothetical individuals (Table 3 ). In this table the parameters of the first, second, and third columns correspond to seropositive individuals who are submitted to three levels (very low/low, medium, and very high) of medication potency and adhesion [11]. The output values of the fuzzy rule-based system are shown in Table 4 . The first line shows the percentage of the infected CD4+ T lymphocytes and the second shows the period of virus replication for the input values of Table 3 . The simulation is performed in a rectangle with $38 \times 38$ cells with 101 iterations. The choice of the cell grid size is defined based on several experimental runs of the CA varying the number of iterations as well as the initial number of elements (uninfected and infected CD4+ T lymphocytes, free virus particles, and virus-specific CTL) and all the other CA parameters necessary for the simulations. The $38 \times 38$ cell grid with 101 iterations showed the closest agreement to qualitative results found in the literature, as in [19-21]. The
CA model is performed for each individual and the results obtained in the simulation in function of time are presented in Figure 2.

\section{Results and Discussion}

3.1. Estimation and Adjustment of the Infection Rates $\beta(t)$. The infection rate $\beta(t)$ of CD4+ T lymphocyte by HIV is an important parameter for AIDS control in HIV-seropositive individuals; therefore, it is determined for the three individuals under study. This rate is determined using the quotient of the number of infected CD4+ T lymphocytes by the product of uninfected $\mathrm{CD} 4+\mathrm{T}$ lymphocytes and the time variation $(\Delta t)[22]$; that is,

$$
\begin{array}{r}
\beta(t)=\frac{\text { infected CD } 4+\text { T lymphocytes }}{\text { (uninfected CD } 4+\text { T lymphocytes }) \cdot \Delta t} \\
t=0,1, \ldots, 100 .
\end{array}
$$


TABLE 1: Fuzzy rules for the percentage of CD4+ cells that will be infected.

\begin{tabular}{|c|c|c|c|c|c|}
\hline \multirow{2}{*}{ Adhesion (A) } & \multicolumn{5}{|c|}{ Medication potency $(\mathrm{M})$} \\
\hline & Very low & Low & Medium & High & Very high \\
\hline Very low & Very high & Very high & Very high & Very high & Very high \\
\hline Low & High & High & High & High & High \\
\hline Medium & Medium & High & Medium & High & Medium \\
\hline High & Medium & High & Low & Low & Low \\
\hline Very high & Low & Low & Low & Very low & Very low \\
\hline
\end{tabular}

TABLE 2: Fuzzy rules for the period of virus replication.

\begin{tabular}{|c|c|c|c|c|c|}
\hline \multirow{2}{*}{ Adhesion (A) } & \multicolumn{5}{|c|}{ Medication potency $(\mathrm{M})$} \\
\hline & Very low & Low & Medium & High & Very high \\
\hline Very low & Very rapid & Very rapid & Very rapid & Very rapid & Very rapid \\
\hline Low & Rapid & Rapid & Rapid & Rapid & Rapid \\
\hline Medium & Medium & Rapid & Medium & Rapid & Medium \\
\hline High & Medium & Rapid & Medium & Slow & Slow \\
\hline Very high & Rapid & Medium & Medium & Very slow & Very slow \\
\hline
\end{tabular}

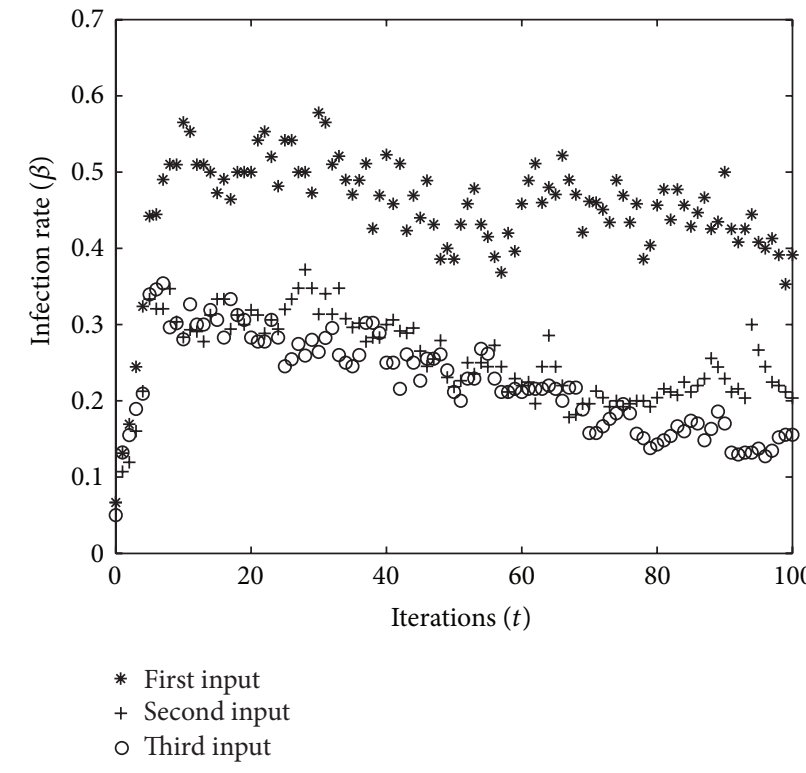

FIGURE 3: CD4+ T lymphocyte infection rates $\beta(t)$ in function of time obtained with the cellular automaton for the three inputs.

TABLE 3: Inputs for the FRBS used in simulation.

\begin{tabular}{lccc}
\hline & First input & Second input & Third input \\
\hline Medication potency & 0.8 & 0.85 & 0.9 \\
Adhesion to treatment & 0.1 & 0.6 & 1 \\
\hline
\end{tabular}

As the result is attained for each iteration, the time variation $(\Delta t)$ is numerically considered to be 1 . Figure 3 presents the infection rate graph in function of time for the three input values. As we observe the graph is descendant, which means that the infection rate of CD4+ T lymphocytes by the virus decreases with time. Such downward curve
TABLE 4: Outputs of the FRBS and input of CA used in simulation.

\begin{tabular}{lccc}
\hline & $\begin{array}{c}\text { First } \\
\text { output-input }\end{array}$ & $\begin{array}{c}\text { Second } \\
\text { output-input }\end{array}$ & $\begin{array}{c}\text { Third } \\
\text { output-input }\end{array}$ \\
\hline $\begin{array}{l}\text { Percentage of infected } \\
\text { CD4+ T lymphocytes }\end{array}$ & 0.85 & 0.55 & 0.1 \\
\hline $\begin{array}{l}\text { Period of virus } \\
\text { replication }\end{array}$ & 6.35 & 10.4 & 16 \\
\hline
\end{tabular}

is expected since we simulate the situation in which an individual receives treatment with antiretrovirals.

With the values obtained (Figure 3 ) we determine three expressions $\beta(t)$ representing the infection rate in function of time $t$ for the three individuals. The values of the six first iterations are not taken into account because they do not represent adequately the biological process to be described. Upon the utilization of the values of the 95 iterations, for each individual, the curve adjustment is obtained by means of the least squares method.

Figure 4 depicts the adjusted points and the graphs of the expression of $\beta(t)$, according to Tables 3 and 4 .

The $\beta(t)$ expressions are

(i) for the first input

$$
\beta(t)=0.2703 e^{-0.005 t}+0.2499 ;
$$

(ii) for the second input

$$
\beta(t)=0.333 e^{-0.005527 t} ;
$$

(iii) for the third input

$$
\beta(t)=0.335 e^{-0.008937 t} .
$$

Figures 3 and 4 present decreasing CD4+ T lymphocyte infection rates in function of time. The determination 


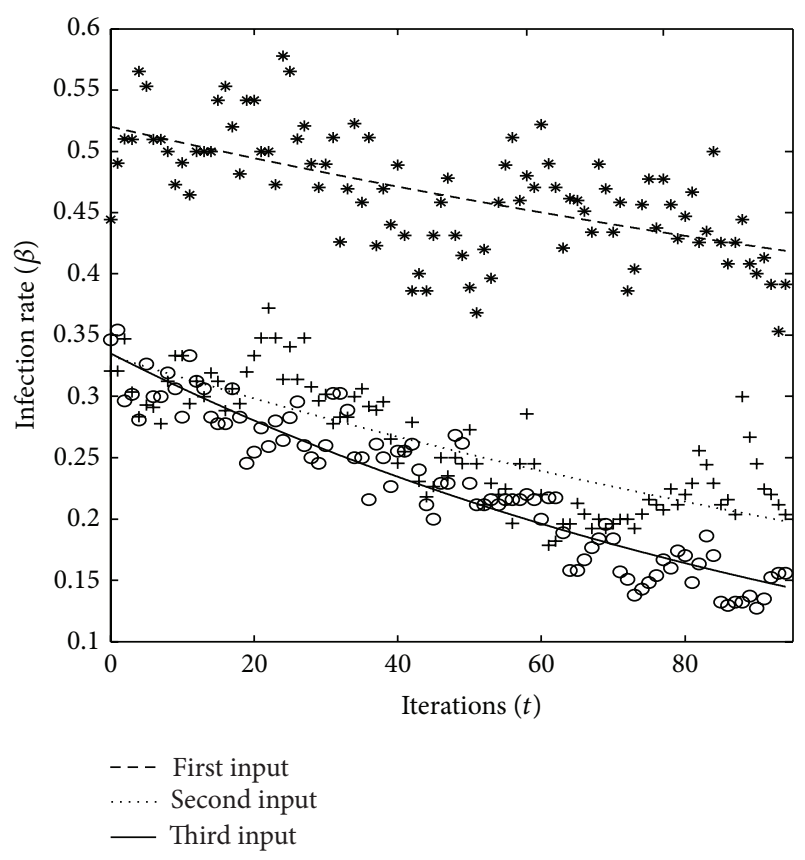

Figure 4: Adjusted CD4+ T lymphocyte infection rates in function of time for the three inputs.

coefficients $\left(R^{2}\right)$ for the adjustments are, respectively, 0.3836 , 0.6333 , and 0.8809 for (5), (6), and (7).

In the next subsection we describe the other parameters obtained with the CA to find the solution of the model with time delay (3) for the three individuals studied.

3.2. Solution for the Delay-Differential Equations Model. We consider the parameters of system (3), that is, the rates, as

$$
\text { rate }=\frac{1}{\text { time }}
$$

where time in the cellular automaton is the number of iterations. The rate values are

(i) $r=20$, which is the constant influx of uninfected cells as produced in the human body which are randomly placed at each iteration in the CA;

(ii) $a=1 / 4$, which is the mortality rate of uninfected cells, as the number of iterations established for death of uninfected cells is 4 ;

(iii) $b=1 / 5$, which is the mortality rate of infected cells, as the number of iterations established for death of infected cells is 5;

(iv) $p=0.4$, which is the death rate of infected cells due to CTL bindings, as a binding is not necessarily successful;

(v) $s=1 / 2$, which is the mortality rate of HIV, as the number of iterations for HIV death is 2;

(vi) $c=1 / 14$, which is the reproduction rate of CTLs, as the number of iterations for CTL reproduction is 14; (vii) $d=1 / 15$, which is the rate of CTL mortality, as the number of iterations for death of CTLs is 15 .

We obtain also the rate $\tilde{a}$ which is the rate of infected cells that have not yet produced virus. In the CA we counted all infected cells that died but did not produce virus at each iteration and then we calculated the average of the counts $(\tilde{a}=0.0825)$. The time-dependent vector $k(t)$ represents the reproduction of infected cells obtained in the automaton, estimated through the direct count of the number of newly reproduced infected cells at each iteration $t$. This vector varies from individual to individual in conformity with medication potency and treatment adhesion. The parameter $\beta(t-\tau)$ represents the infection rate for a previous time with delay $\tau$ and so it is necessary to obtain the adjustment of the curve $\beta(t)$ for each individual. For the numeric solution of system (3), represented in Figure 5, we consider the adjustment (6) that represents the seropositive individual receiving medium potency medication and medium treatment adhesion, the delay $\tau=0.5$, and the rates defined previously. The initial conditions are $n=0.99, i=0.01, v=0.1$, and $z=0.01$. The software Matlab was used to obtain the numerical solution for the system.

We employed Matlab routine dde23 that tracks discontinuities and integrates with the explicit Runge-Kutta method where the error is given $O\left(h^{3}\right)$ per step and $O\left(h^{2}\right)$ in total and interpolant of ode23. It uses iteration to take steps longer than the lags. ode23 is also an implementation of an explicit Runge-Kutta method.

In Figure 5 we observe that, as time elapses, the uninfected cells and the CTLs increase up to a steady number and the viral load and the infected cells decrease drastically. This is the main goal of any therapy using antiretroviral treatment, that is, to increase the number of CD4+ T lymphocytes and to decrease the viral load.

\subsection{Solution for the Fuzzy Delay-Differential Equations Model.} We regard both pharmacological and intracellular delays as a unique uncertain parameter. Mittler et al. (1998) consider the delay distribution as a gamma probability distribution which may mimic a variety of biologically possible delays. In the present paper the delay is characterized by a distribution of possibilities that is modeled mathematically by means of a fuzzy number. We consider the delay $\tau$ as a fuzzy number as depicted in Figure 6 using the interval $[0.08,1]$ as the support of $\tau$. This interval is chosen based on [7] in which experimentally $\tau$ is uncertain and assumes the values of 0.08 days (approximately $2 \mathrm{~h}$ ), 0.5 days (12 h), and 1 day ( $24 \mathrm{~h}$ ). We utilize Zadeh's Extension Principle in order to obtain the nonlinear system solution with fuzzy delay (3). In system (3) the delay only appears in the equation of infected cells. At each instant $t$ that principle is applied to the numerical solution of the equation of infected cells with delay $\tau$.

Figure 7 presents the solution of the equation of infected cells. Other solutions of the equations for system (3) with fuzzy delay present similar behavior to the ones with a constant delay, that is, nonfuzzy, as in Figure 5. We observe that, in the central region of the graph of infected cells, the membership degree is close to 1 ; that is, the darker it is, the 

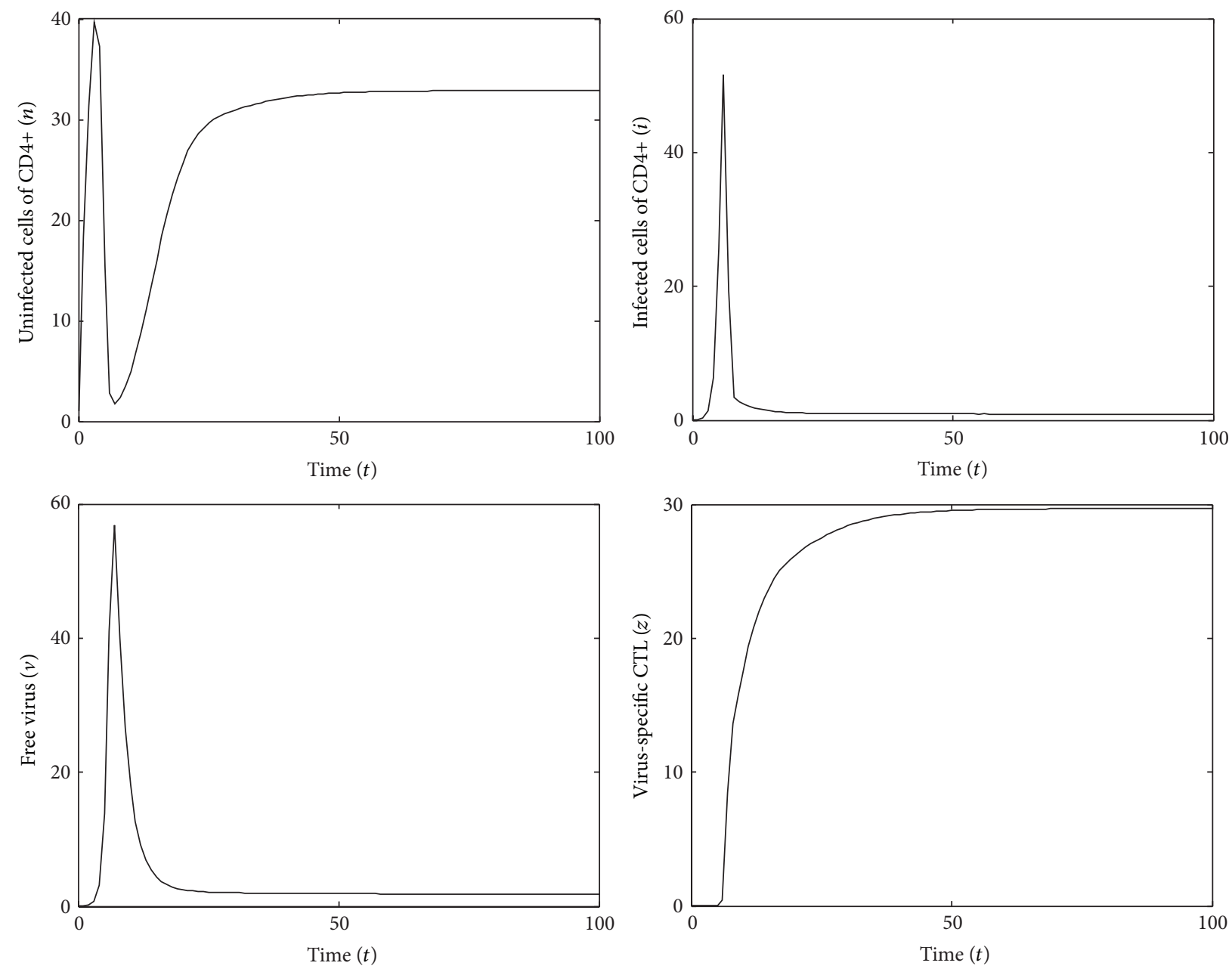

FIGURE 5: Nonlinear numeric solution of system (3) with delay.

smaller is the degree. This region is the best one to represent the biological phenomena as far as credibility is concerned.

In order to represent the evolution of infected cells in this model through a deterministic curve, the graph in Figure 7 must be defuzzified. Figure 8 presents the defuzzified solution for the infected cells, which turns out to be the average fuzzy solution [4].

The difference between the classic deterministic model and the fuzzy model is that, in the former, the uncertainties are excluded at the beginning while in the latter the uncertainties evolve in time and the defuzzification occurs at the moment of interest. The center of gravity method is utilized for the defuzzification at each instant $t$ as described in [9]. Figure 9 depicts the defuzzified solutions for the three HIVseropositive individuals.

With the defuzzified solution we obtain the maximum values of infected cells for hypothetical individuals that received low, medium, and high potency medication and treatment adhesion, respectively, 44.9514, 44.0362, and 0.3523 . These results are in accordance qualitatively with what would be expected, since individuals who received the weakest treatment present the highest number of infected cells, even higher than those of individuals who received the best treatment.

This research, although of computational nature, may collaborate for the better understanding of HIV dynamics as a complement of biological techniques like HIV spread, two- and three-dimensional cell culture systems, imaging approaches to HIV spread visualization, and others as referred by Fackler et al. (2014) [23].

\section{Conclusion}

The cellular automaton was of paramount importance for obtaining the $\mathrm{CD} 4+\mathrm{T}$ lymphocyte infection rate $(\beta(t))$ by HIV as well as the other parameters necessary to solve the delay-differential equation system. The behaviors of the HIV and the uninfected CD4+ T lymphocytes agree with the respective behaviors reported in [19-21]. In general, the $\mathrm{CD} 4+\mathrm{T}$ lymphocytes infection rate is a parameter that medical science faces difficulty to obtain. Moreover, upon the medical point of view, it might be important to permit the uncertainty to evolve in time so that answers for those biological issues can be answered. Upon utilization of the 


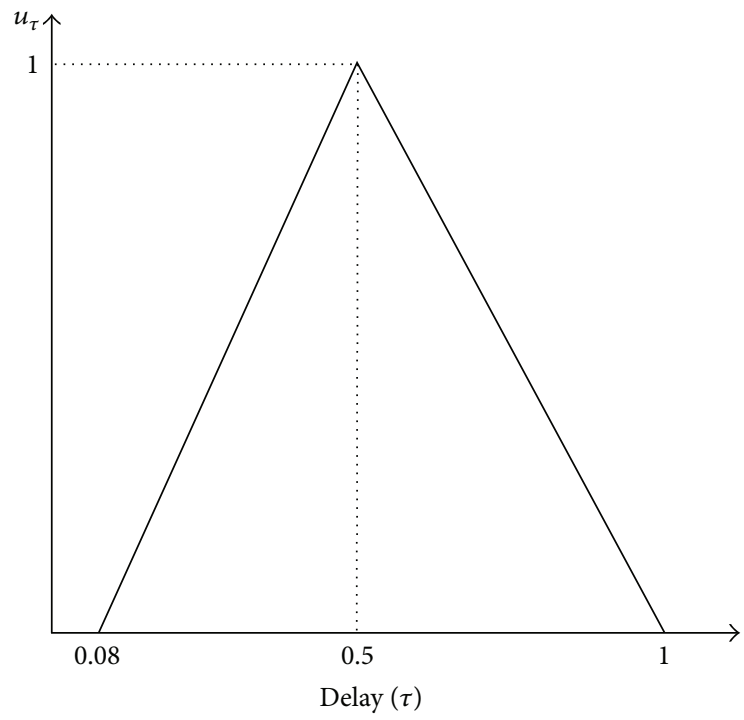

FIgURE 6: Distribution of possibilities for the delay $(\tau)$.

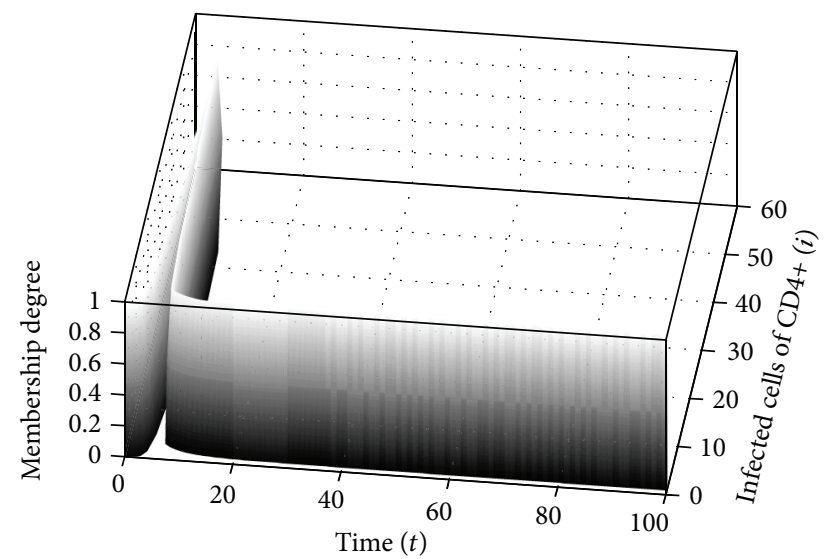

FIgURE 7: Fuzzy solution for infected CD4+ T lymphocytes of system (3).

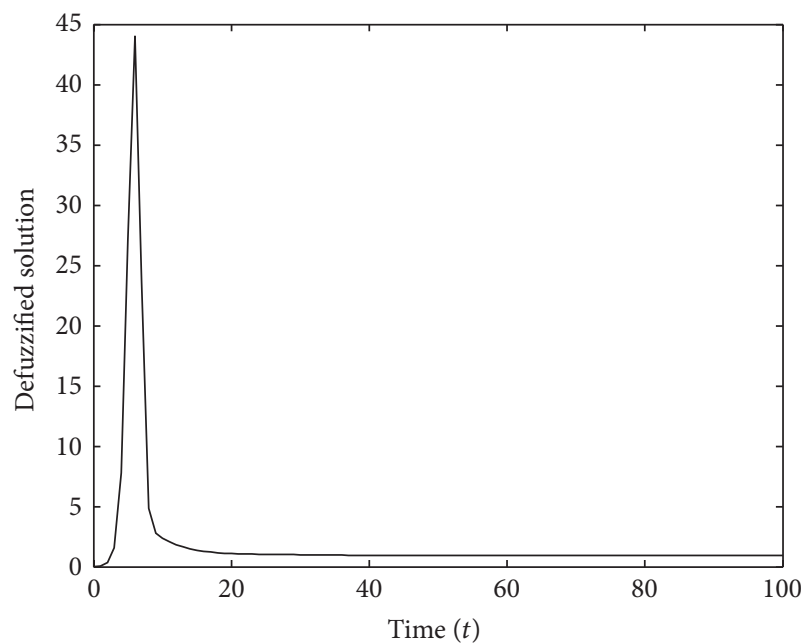

FIGURE 8: Defuzzified solution for infected CD4+ T lymphocytes of system (3).

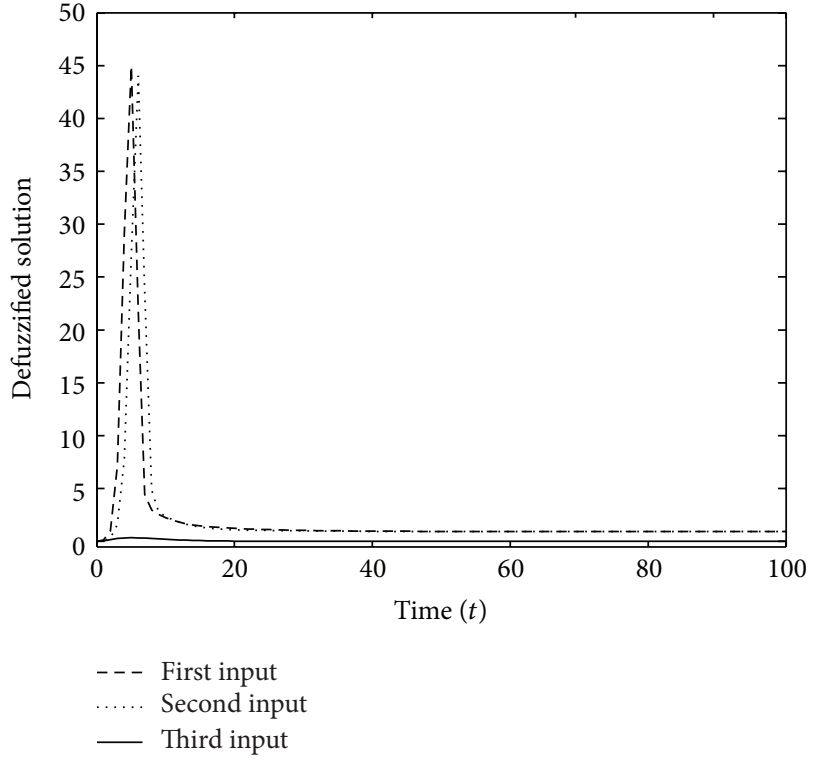

FIgURE 9: Defuzzified solution for infected CD4+ T lymphocytes of system (3) for the three individuals.

fuzzy delay model we present an approach to the estimation of CD4+ $\mathrm{T}$ lymphocyte infection rates that incorporates possible uncertainties inherent of such important health issue.

\section{Conflict of Interests}

The authors declare that there is no conflict of interests regarding the publication of this paper.

\section{Acknowledgment}

The first and third authors acknowledge CNPq, the Brazilian National Research Council, for financial support (Process 477918/2010-7 and Process 305862/2013-8, resp.).

\section{References}

[1] UNAIDS, http://www.unaids.org.

[2] Ministry of Health, (Portuguese) http://www.aids.gov.br.

[3] R. M. Jafelice, L. C. de Barros, R. C. Bassanezi, and F. Gomide, "Fuzzy modeling in symptomatic HIV virus infected population," Bulletin of Mathematical Biology, vol. 66, no. 6, pp. 15971620, 2004.

[4] R. M. Jafelice, L. C. de Barros, R. C. Bassanezi, and F. Gomide, "Methodology to determine the evolution of asymptotic HIV population using fuzzy set theory," International Journal of Uncertainty, Fuzziness and Knowledge-Based Systems, vol. 13, no. 1, pp. 39-58, 2005.

[5] N. Ortega, Aplicação da Teoria de Lógica Fuzzy a Problemas da Biomedicina [Ph.D. thesis], Instituto de Física-USP, São Paulo, Brazil, 2001, (Portuguese).

[6] H. Zarei, A. V. Kamyad, and A. A. Heydari, "Fuzzy modeling and control of HIV infection," Computational and Mathematical Methods in Medicine, vol. 2012, Article ID 893474, 17 pages, 2012. 
[7] A. V. M. Herz, S. Bonhoeffer, R. M. Anderson, R. M. May, and M. A. Nowak, "Viral dynamics in vivo:limitations on estimates of intracellular delay and virus decay (medical sciences)," Proceedings of the National Academy of Sciences of the United States of America, vol. 93, no. 14, pp. 7247-7251, 1996.

[8] J. E. Mittler, B. Sulzer, A. U. Neumann, and A. S. Perelson, "Influence of delayed viral production on viral dynamics in HIV-1 infected patients," Mathematical Biosciences, vol. 152, no. 2, pp. 143-163, 1998.

[9] R. M. Jafelice, L. C. Barros, and R. C. Bassanezi, "Study of the dynamics of HIV under treatment considering fuzzy delay," Computational \& Applied Mathematics, vol. 33, no. 1, pp. 45-61, 2014.

[10] G. B. Ermentrout and L. Edelstein-Keshet, "Cellular automata approaches to biological modeling," Journal of Theoretical Biology, vol. 160, no. 1, pp. 97-133, 1993.

[11] R. M. Jafelice, B. F. Z. Bechara, L. C. Barros, R. C. Bassanezi, and F. Gomide, "Cellular automata with fuzzy parameters in microscopic study of positive HIV individuals," Mathematical and Computer Modelling, vol. 50, no. 1-2, pp. 32-44, 2009.

[12] L. A. Zadeh, "Fuzzy sets," Information and Computation, vol. 8, pp. 338-353, 1965.

[13] W. Pedrycz and F. Gomide, An Introduction to Fuzzy Sets: Analysis and Design, Massachusetts Institute of Technology, 1998.

[14] E. Massad, N. Ortega, L. C. Barros, and C. Struchiner, Fuzzy Logic in Action: Applications in Epidemiology and Beyond, vol. 232 of Studies in Fuzziness and Soft Computing, Springer, Berlin, Germany, 2008.

[15] M. A. Nowak and C. R. M. Bangham, "Population dynamics of immune responses to persistent viruses," Science, vol. 272, no. 5258, pp. 74-79, 1996.

[16] E. F. Moore, "Machine models of self-reproduction," Proceedings of Symposia in Applied Mathematics, vol. 14, pp. 17-34, 1962.

[17] C. Renning, "Collective behaviour: emergent dynamics in populations of interacting agents," in Proceedings of the Artificial Life Seminar, 1999-2000.

[18] H. Ying, F. Lin, X. Luan et al., "A fuzzy discrete event system with self-learning capability for HIV/AIDS treatment regimen selection," in Proceedings of the Annual Meeting of the North American Fuzzy Information Processing Society (NAFIPS '05), pp. 820-824, Detroit, Mich, USA, June 2005.

[19] R. A. Filter, X. Xia, and C. M. Gray, "Dynamic HIV/AIDS parameter estimation with application to a vaccine readiness study in Southern Africa," IEEE Transactions on Biomedical Engineering, vol. 52, no. 5, pp. 784-791, 2005.

[20] J. Guedj, R. Thiébaut, and D. Commenges, "Practical identifiability of HIV dynamics models," Bulletin of Mathematical Biology, vol. 69, no. 8, pp. 2493-2513, 2007.

[21] D. A. Ouattara, M. J. Mhawej, and C. H. Moog, “Clinical tests of therapeutical failures based on mathematical modeling of the HIV infection," IEEE Transactions on Automatic Control, vol. 53, pp. 230-241, 2008.

[22] A. Freitas, Avaliação de novas estratégias vacinais contra a coqueluche no estado de São Paulo [M.S. thesis], Faculdade de Saúde Pública, Universidade de São Paulo, São Paulo, Brazil, 2008.

[23] O. T. Fackler, T. T. Murooka, A. Imle, and T. R. Mempel, "Adding new dimensions: towards an integrative understanding of HIV1 spread," Nature Reviews Microbiology, vol. 12, no. 8, pp. 563574, 2014. 


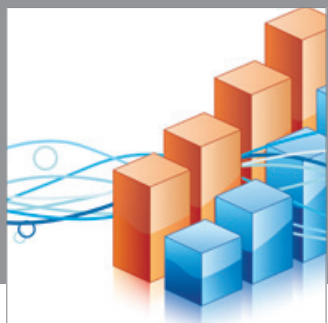

Advances in

Operations Research

mansans

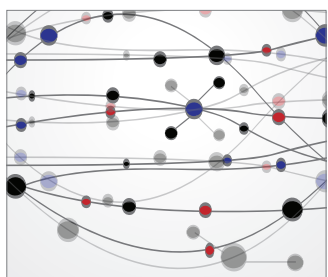

The Scientific World Journal
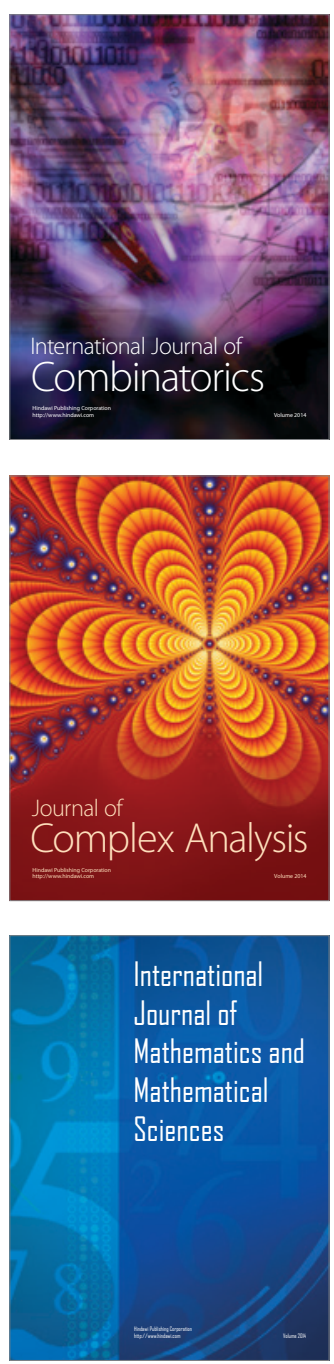
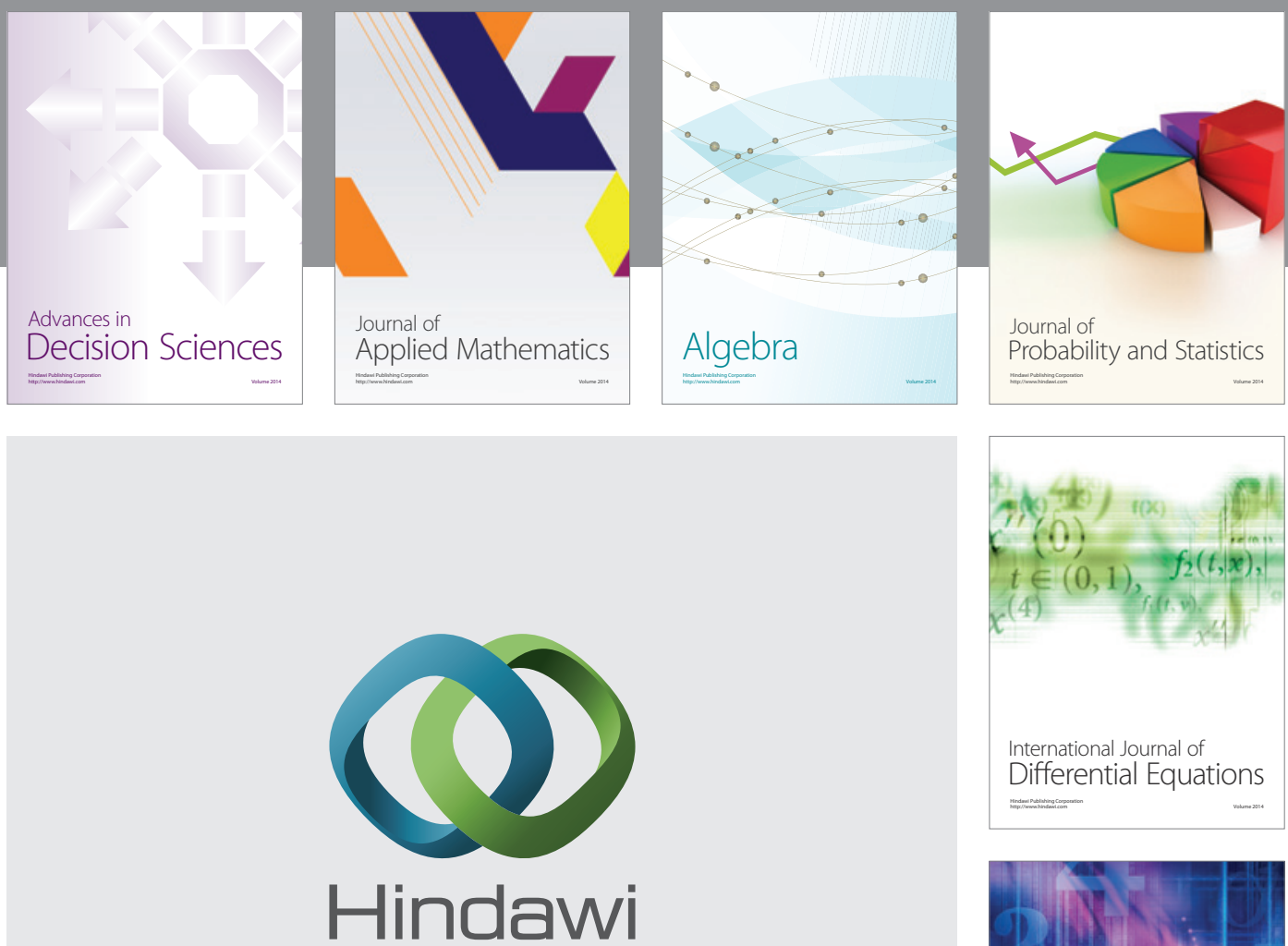

Submit your manuscripts at http://www.hindawi.com
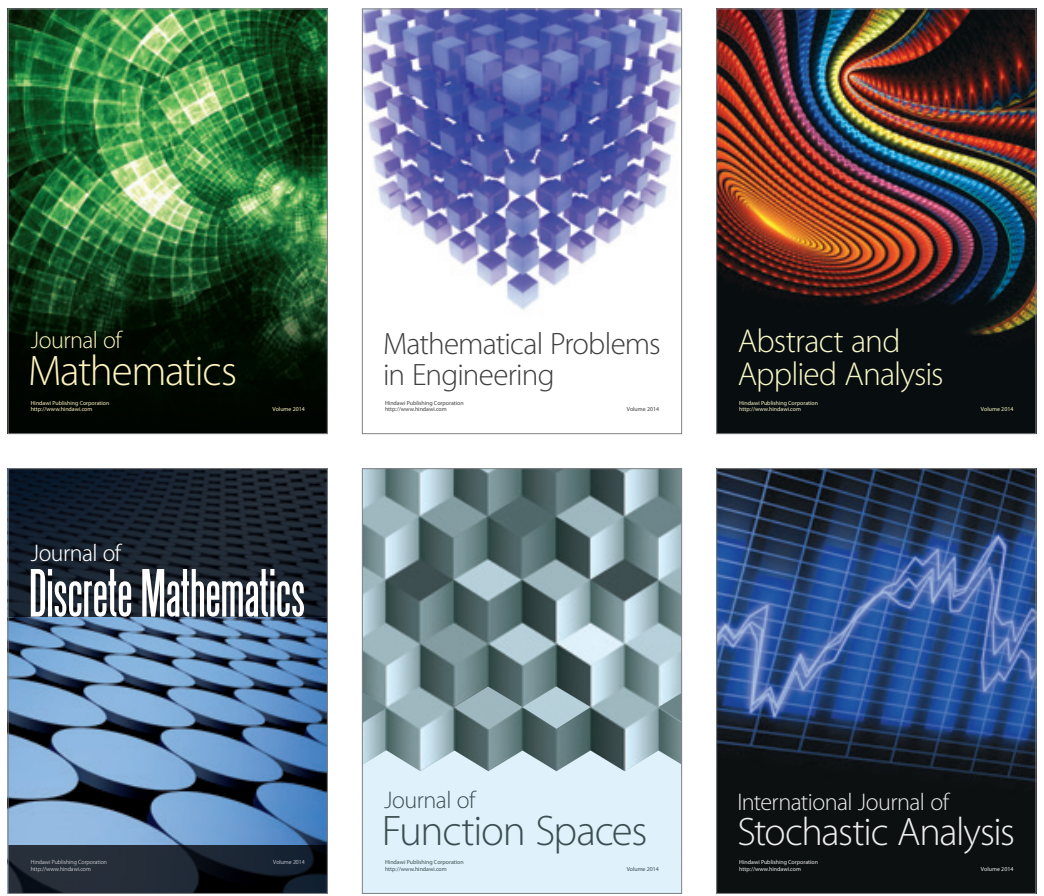

Journal of

Function Spaces

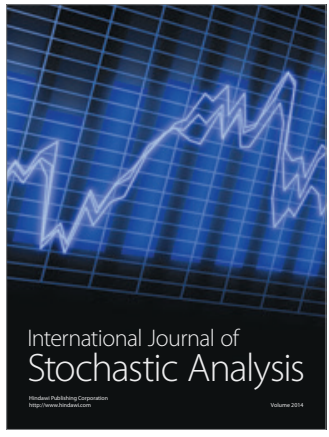

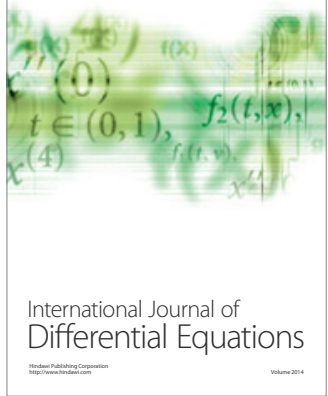
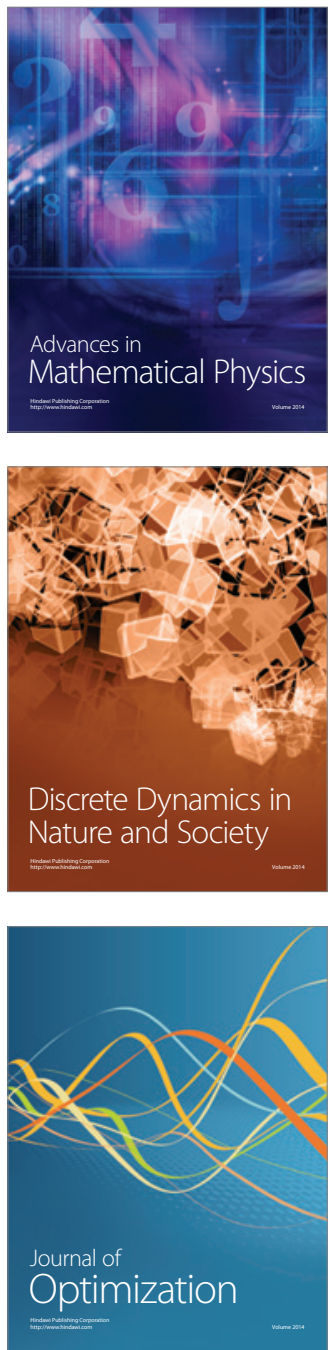